\title{
Prospective evaluation of a prognostic index for intrinsic supratentorial tumours
}

\author{
J L Hutton, D F Smith, D W Chadwick
}

\begin{abstract}
A prognostic index for patients with intrinsic supratentorial tumours based on prebiopsy findings was proposed on the basis of data bases of patients presenting between 1975 and 1989. The value of this index is confirmed from data on 76 patients who presented during 1991. Patients classified by the index as having a poor prognosis had a median survival of six months, and those classified as having a good prognosis had a median survival of five years. The expected duration of survival, allowing for this initial prognosis, also depended on whether a patient received resective surgery, radiotherapy, or steroids. Histological grade provided no additional information on survival.
\end{abstract}

(F Neurol Neurosurg Psychiatry 1995;59:92-94)

Keywords: brain tumour; prognosis; evaluation of prognostic index

The prognosis of patients with intrinsic supratentorial tumours has been shown to depend strongly on the health of the patients at presentation and on treatment. It is useful for clinicians and patients to have an estimate of how good a particular patient's prognosis is. On the basis of a retrospective study of 560 patients who attended Walton Hospital between 1975 and $1989,{ }^{1}$ we proposed two prognostic indices based on age, symptoms, and CT findings. ${ }^{2}$ An initial index used the duration of symptoms as well as age, whether the presenting symptom was epilepsy, presence of focal signs, and cyst on CT. A study of the reliability of the initial index resulted in our proposing a modified index in which the duration of symptoms was replaced by the presence of contrast enhancement, diffuse low density and calcification on CT, and mental signs. Table 1 shows the scores assigned to each variable. Presenting with epilepsy, young age, the absence of focal signs, mental signs, and contrast enhancement on CT and the presence of cystic change, diffuse low density, and calcification on CT were associated with a good prognosis. Patients with a total score of 16 or less were defined as having a good prognosis. The median survival of patients with good prog- noses was 27 months, and for the remaining patients it was three months.

Although the index performed well on the database from which it was derived, its performance needed to be externally validated. This paper presents the results of an evaluation of the proposed index from data on a group of patients with brain tumours who attended Walton Hospital during 1991. A simple alternative method of deciding a patient's prognosis is to classify patients whose first symptom is epilepsy as having good prognosis. The performance of this criterion and the usefulness of histological grade are also evaluated and compared with that of the index.

\section{Patients and methods}

Seventy six patients who attended Walton Hospital with intrinsic supratentorial tumours during 1991 were followed up for at least seven months or until death. Some patients had had a diagnosis before 1991 . The date of death was known for 50 patients. The remaining patients were known to have lived between seven and 153 months after diagnosis.

The performance of the index in defining two prognostic groups (hereafter simply referred to as "good on index"), whether the first symptom was epilepsy (hereafter simply referred to as "epilepsy"), and the histological

Table 1 Scores assigned to variables for prognostic index

\begin{tabular}{llr}
\hline Variable & Categories & Score \\
\hline Age (y) & $<45$ & 0 \\
& $45-59$ & 5 \\
& $>59$ & 10 \\
Focal signs: any definite clinical signs & & \\
locating a lesion to one part of a & & \\
cerebral hemisphere-for example, & Absent & 0 \\
hemiparesis & Present & 5 \\
First sympton & Not epilepsy & 7 \\
Cyst on CT: a circular low density & Epilepsy & 0 \\
area before enhancement, with & Absent & 0 \\
clear cut margins & Present & -4 \\
Contrast enhancement on CT & Absent & 0 \\
Diffuse low density on CT: diffuse, & Present & 7 \\
poorly demarcated low density on & Absent & 0 \\
$\quad$ CT without contrast enhancement & Present & -3 \\
Calcification on CT & Absent & 0 \\
Mental signs: any clinical sign of & Present & -4 \\
$\quad$ confusion, altered awareness, or & Absent & 0 \\
personality change & Present & 3 \\
\hline
\end{tabular}


Figure 1 Survival of patients with intrinsic supratentorial tumours in prognostic groups defined by index -..-good prognosis; - poor prognosis.

Figure 2 Survival of patients with intrinsic supratentorial tumours in by first symptom. epilepsy; - - - - not epilepsy. prognostic groups defined
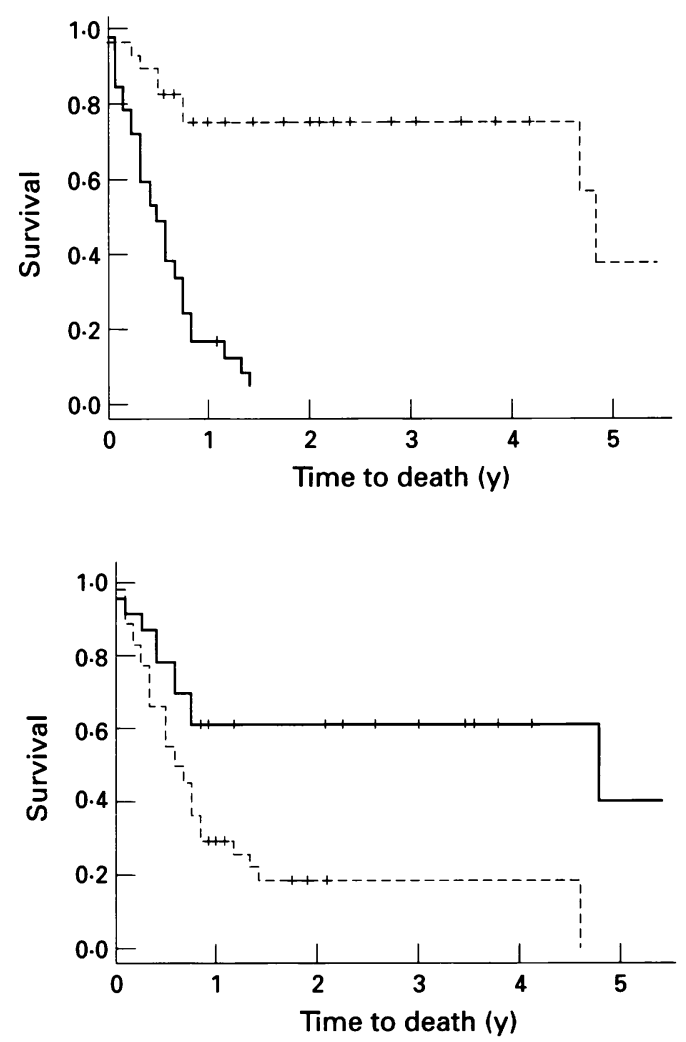

grade (grouped as I/II and III/IV), in predicting survival were evaluated by comparing the difference in the survival of the groups with good and poor prognoses. The comparison was made with Kaplan-Meier estimates of the survival curves and the associated log rank statistics. It is possible that good on index

Table 2 Relation between first symptom and index group

\begin{tabular}{llll}
\hline & \multicolumn{3}{l}{ Index group } \\
\cline { 2 - 4 } First symptom & Good & Poor & Total \\
\hline Epilepsy & 18 & 5 & $23(38)$ \\
Not epilepsy & 11 & 42 & $53(62)$ \\
Total & $29(30)$ & $47(70)$ & 78 \\
\hline
\end{tabular}

Numbers in parentheses are percentages.

Table 3 Resective surgery, radiotherapy, or steroids, and histological grade for patients classified by index or first symptom

\begin{tabular}{llllll}
\hline & \multicolumn{2}{l}{ Index group } & & \multicolumn{2}{l}{ First symptom } \\
\cline { 2 - 3 } & Good & Poor & & Epilepsy & Not epilepsy \\
\hline Resective surgery performed & $12(41)$ & $13(28)$ & & $9(39)$ & $16(30)$ \\
Steroids given & $18(62)$ & $47(100)$ & & $14(61)$ & $51(96)$ \\
Radiotherapy & $17(59)$ & $33(70)$ & & $14(61)$ & $36(68)$ \\
Grade III or IV & $10(34)$ & $19(40)$ & & $10(43)$ & $19(35)$ \\
Total & 29 & 47 & & 23 & 53 \\
\hline
\end{tabular}

Numbers in parentheses are percentages.

Table 4 Estimated effects of resective surgery, radiotherapy, steroids, and index, using a proportional hazards model

\begin{tabular}{llllc}
\hline Variable & Coefficient & $\begin{array}{l}\text { Standard } \\
\text { error }\end{array}$ & Probability & Risk ratio \\
\hline Good prognosis on index & -1.64 & 0.45 & 0.0003 & 0.2 \\
Patient received steroids & 2.54 & 1.11 & 0.0224 & 12.7 \\
Resective surgery performed & -0.99 & 0.37 & 0.0072 & 0.4 \\
Patient received radiotherapy & -1.42 & 0.35 & 0.0001 & 0.2 \\
\hline
\end{tabular}

and epilepsy might predict which patients will receive resective surgery, radiotherapy, and steroids. To investigate which factors provide the best explanation of differences in survival, a proportional hazards regression $\operatorname{model}^{3}$ was fitted with histological grade, resective surgery, radiotherapy, and steroids as well as good on index or epilepsy as explanatory variables.

\section{Results}

Twenty nine patients were good on index and nine $(31 \%)$ of them were known to have died. Of those with a poor prognosis, $41(87 \%)$ had died. Twenty three patients presented with epilepsy, of whom $10(43 \%)$ were known to have died. Forty $(75 \%)$ of the other patients had died. These two classifications of prognosis differed for 16 patients (table 2). The index identified 11 patients who did not present with epilepsy as having a good prognosis, and five patients with epilepsy as having a poor prognosis. Both good on index and epilepsy defined groups with very different survival experience (figs 1 and 2 ). The separation between patients with good and poor prognosis was greater for the index groups. The log rank $\chi^{2}$ statistics were $9.2(P<0.01)$ for epilepsy and 29.3 $(P<0.01)$ for the index, showing that the index discriminates more effectively than epilepsy. (Using the original index yielded a log rank $\chi^{2}$ statistic of 14.1 ( $P<0.01)$.) Histological grade did not yield groups with different survival: the log rank $\chi^{2}$ was $0.69(P>0.40)$. The median survival for patients presenting with epilepsy was nearly five years, and only seven months for the others. The median for good on index was also nearly five years, and six months for poor on index.

Table 3 shows that the better survival of patients with a good prognosis might in part be attributed to treatment. More of those good on index or with epilepsy received resective surgery, but fewer received only radiotherapy or steroids. Histological grade was not associated with good on index or epilepsy; roughly $40 \%$ of patients had tumours graded III or IV.

Table 4 gives estimates of the combined effects of type of treatment, steroids, and good on index. Use of steroids was associated with an increased relative risk of death of 13 . Those who were poor on index had five times the relative risk of the good on index group. Those who did not receive resective surgery and radiotherapy had relative risks 2.5 and five times higher than treated patients. A proportional hazards model fitted with epilepsy, resective surgery, radiotherapy, and steroids as variables showed that knowledge of whether the presenting symptom was epilepsy is not informative once treatment is taken into account. A proportional hazards model fitted with histological grade and good on index showed that grade adds no information on survival once good on index is taken into account (histological grade, $\mathrm{P}=0.4$; good on index, $P<0.01$ ). 


\section{Discussion}

This study has shown that the prognostic index proposed with information available before biopsy can be used to decide which patients have a good prognosis. The median survival for patients with a score of 16 or less on the index was almost five years, by contrast with a median of only six months for the remaining patients. Three quarters of those with poor prognoses die before a quarter of those with good prognoses die. A greater proportion of patients who had a poor prognosis on the index received steroids and radiotherapy, and fewer had resective surgery. When the use of steroids, resective surgery, and radiotherapy is taken into account, a substantial part of the variation in survival is still attributed to the classification of patients using the index.

A simpler indicator of a good prognosishaving epilepsy as the first symptom of a tumour-is also effective in defining a group with good survival. It discriminates less well than the index, however, and has no explanatory value after allowing for treatment and steroids. The survival of patients with histological grades I and II did not differ from that of patients with grades III and IV. This suggests that the grading of tumours might not be accurate in determining prognosis.

The prognostic index developed by the Medical Research Council Brain Tumour Working Party (MRCBTWP) ${ }^{4}$ for high grade malignant glioma uses similar age categories to those of our index. Our original index used the duration of fits, as does the MRCBTWP index, but our revised index replaced this with mental signs and CT findings. The original index performed slightly better than epilepsy, but less well than the revised index. The MRCBTWP index uses extent of neurosurgery and clinical performance, whereas our index uses only prebiopsy data. The median survival of the group with poor prognosis defined by our index is compatible with the survival reported in the MRC trial of radiotherapy doses. ${ }^{5}$ In both cases, the prognostic index provides information about survival additional to the effect of treatment. More detailed classification of prognosis could be made with our score, as is done with the MRCBTWP index. This could be useful in responding to enquiries by patients about the seriousness of their illness.

The index provides good discrimination of patients with intrinsic supratentorial tumours, and is able to identify a larger number of patients as having a good prognosis than epilepsy alone. These results justify the use of the proposed index as a criterion for entry to a clinical trial. There is still no consensus about whether to intervene surgically as soon as a low grade glioma is suspected. ${ }^{6-8} \mathrm{It}$ is clearly difficult to carry out a clinical trial to compare conservative treatment with early surgical intervention, as many patients and physicians are reluctant to enter such a trial. Although Shaw ${ }^{7}$ claims that there is evidence to justify such reluctance, he does not discuss adequately the biases inherent in retrospective studies. ${ }^{8}$ There is now some evidence that neither resective surgery nor radiotherapy improve the survival of patients who present with epilepsy. ${ }^{1}$

The results presented here and by the $M C^{5}$ show that a thorough analysis of all retrospective studies of the effects of delayed treatment on the survival of patients with brain tumours ought to take into account the differences between patients who had early surgery and those who did not. This could be done with the prognostic index described here. Until such a thorough analysis is done good evidence in favour of either management strategy is likely to remain lacking.

1 Smith DF, Hutton JL, Sandemann D, Foy PM, Shaw MDM, Williams IR, et al. The prognosis of primary intracerebral tumours presenting with epilepsy: the outcome of medical and surgical management. $f$ Neurol Neurosurg Psychiatry 1991;54:915-20.

2 Hutton JL, Smith DF, Sandemann D, Foy PM, Shaw MDM, Williams IR, et al. Development of a prognostic index for use in a trial of medical and surgical management of primary intracellular tumours. $f$ Neuro Neurosurg Psychiatry 1992;55:271-4.

3 Tibshirani $R$. A plain man's guide to the proportional hazards model. Clin Invest Med 1982;5:63-8.

MRC Brain Tumour Working Party Prognostic factors for malignant glioma: development of a prognostic index. F Neurooncol 1990;9:47-55.

5 Bleehen NM, Stenning SP. A Medical Research Council trial of two radiotherapy doses in the treatment of grades 3 and 4 astrocytoma. $\mathrm{Br} \mathcal{F}$ Cancer 1991;64 769-74.

Recht LD, Lew R, Smith TW. Suspected low-grade glioma: is deferring treatment safe? Ann Neurol 1992,

Shaw EG. Low-grade gliomas: to treat or not to treat? Arch Neurol 1990;47:1138-9. Cairncross JG, Laperriere NJ. Low-grade gliomas: to treat
or not to treat? A reply. Arch Neurol 1990;47:1139-40. 